\title{
INFLUENCIA DEL NIVEL DE FERMENTACIÓN DEL VINO BASE SOBRE ALGUNOS COMPUESTOS VOLÁTILES DEL PISCO PERUANO DE UVA ITALIA
}

\author{
Yasser Hidalgo*a $^{*}$, Beatriz Hatta ${ }^{\mathrm{a}}$, Juan Carlos Palma ${ }^{\mathrm{b}}$
}

\begin{abstract}
RESUMEN
Se investigó la influencia del nivel de fermentación del vino base sobre algunos compuestos volátiles en el pisco, tales como: ácido acético, alcoholes superiores, ésteres, acetaldehído y metanol. Para evaluar el efecto del nivel de fermentación, durante esta etapa se obtuvieron cinco muestras de vino base con diferentes densidades de corte; para luego ser de inmediato destiladas. Los resultados indicaron que los piscos provenientes de vinos base con menores niveles de fermentación, expresados con mayores densidades de corte $(990 ; 1000 ; 1010 ; 1025$ y $1040 \mathrm{~g} / \mathrm{L})$, presentan mayores contenidos de ácido acético $(0.15,0.16,0.17,0.17$ y 0.18 $\mathrm{g} / \mathrm{L})$, propanol $(72.15,76.33,82.25,89.86$ y $95.50 \mathrm{mg} / 100 \mathrm{~mL}$ alcohol anhidro), acetato de etilo (22.00, 30.72, 34.26, 39.06 y $49.68 \mathrm{mg} / 100 \mathrm{~mL}$ alcohol anhidro), acetaldehído (22.71, $40.52,48.69,58.51$ y $78.62 \mathrm{mg} / 100 \mathrm{~mL}$ alcohol anhidro) y metanol $(39.22,40.27,43.33$, 51.30 y $57.95 \mathrm{mg} / 100 \mathrm{~mL}$ alcohol anhidro); y menores contenidos de alcohol iso/ter-amílico $(122.83,118.98,115.78,102.88$ y $100.11 \mathrm{mg} / 100 \mathrm{~mL}$ alcohol anhidro).
\end{abstract}

Palabras clave: Destilados, pisco, pisco mosto verde, nivel de fermentación, compuestos volátiles.

\section{INFLUENCE OF LEVEL FERMENTATION OF BASE WINE IN SOME VOLATILE COMPOUNDS OF PERUVIAN ITALIA GRAPE PISCO}

\begin{abstract}
The influence of level fermentation of base wine in some volatile compounds in Pisco obtained therefrom were studied, such as: acetic acid, higher alcohols, esters, acetaldehyde and methanol. In order to evaluate the effect of level fermentation, during this stage five base wine samples with five different cut densities were obtained; to be distilled straight away. The results indicated that piscos obtained from musts with lower fermentation levels, expressed by higher cut densities $(990 ; 1,000 ; 1,010 ; 1,025$ and $1,040 \mathrm{~g} / \mathrm{L})$, show higher acetic acid

\footnotetext{
${ }^{1}$ Facultad de Industrias Alimentarias, Universidad Nacional Agraria La Molina. Av. La Universidad s/n, Lima 12, Perú.

*yasser_hds@yahoo.com

b Facultad de Ciencias - Dpto. de Química, Universidad Nacional Agraria La Molina, Av. La Universidad s/n, Lima 12, Perú.
} 
$(0.15,0.16,0.17,0.17$ and $0.18 \mathrm{~g} / \mathrm{L})$, propanol $(72.15,76.33,82.25,89.86$ and $95.50 \mathrm{mg} / 100$ $\mathrm{mL}$ anhydrous alcohol), ethyl acetate $(22.00,30.72,34.26,39.06$ and $49.68 \mathrm{mg} / 100 \mathrm{~mL}$ anhydrous alcohol), acetaldehyde (22.71, 40.52, 48.69, 58.51 y $78.62 \mathrm{mg} / 100 \mathrm{~mL}$ anhydrous alcohol) and methanol $(39.22,40.27,43.33,51.30$ and $57.95 \mathrm{mg} / 100 \mathrm{~mL}$ anhydrous alcohol) contents; and lower iso/ter-amyl alcohol $(122.83,118.98,115.78,102.88$ and $100.11 \mathrm{mg} / 100$ $\mathrm{mL}$ anhydrous alcohol).contents.

Key words: Distillates, pisco, green must pisco, fermentation level, volatile compounds.

\section{INTRODUCCIÓN}

El pisco peruano es el aguardiente obtenido exclusivamente por destilación de mostos frescos de "uvas pisqueras" recientemente fermentados, utilizando métodos que mantengan el principio tradicional de calidad establecido en las zonas de producción reconocidas ${ }^{1}$.

Desde su aparición en el mercado, la elaboración de pisco tipo "Mosto Verde" ha tomado un protagonismo interesante y ha generado diversos comentarios, en su mayoría favorables, ya que, al ser destilado a partir de mostos frescos incompletamente fermentados, le darían a éste ciertas características sensoriales particulares a su estructura y cierta sensación "aterciopelada" en boca. Sin embargo, hasta el momento el sustento técnico científico de este tipo ha sido insuficiente.

\section{Materia prima}

Los controles se realizan en primer lugar sobre la uva, que cosechada convenientemente en recipientes no contaminantes como jabas de plástico deberá presentar un buen estado sanitario, exenta de restos de pesticidas o sustancias peligrosas que puedan influenciar el normal desarrollo de la fermentación alcohólica o transformarse en componentes volátiles tóxicos que pasen al destilado ${ }^{2}$.

En el transporte de uvas a granel hacia la bodega, la altura de la masa vitícola almacenada en los recipientes no deberá superar un metro, para evitar altas presiones que rompan los hollejos y que hagan fluir el mosto².

\section{Mosto}

El procesado de la uva, para la obtención del mosto, se debe realizar lo más rápido posible, para evitar la oxidación del mosto. Este proceso deberá hacerse en condiciones higiénicas óptimas para conservar en el mosto las bondades intrínsecas de la uva que luego harán parte de las características cualitativas del destilado².

El prensado tiene por objeto extraer la solución acuosa contenida en las bayas así como ciertos compuestos del hollejo bajo el efecto de la presión. Esta operación se realiza en una prensa, que es en general un cilindro en el interior del cual la vendimia es comprimida, sea por el movimiento de uno o dos platos (prensas mecánicas) o por el inflado de una membrana 
(prensas neumáticas). La presión aplicada debe ser progresiva y alcanzar valores finales bastante elevados pero no demasiado para no romper los raspones, los hollejos y las pepitas ${ }^{3}$.

La fermentación alcohólica del mosto debe realizarse en recipientes que no contaminen el vino base a obtenerse, que al ser un producto orgánico, es perecible ${ }^{2}$.

\section{Fermentación}

El poder reductor producido por la glucólisis, bajo la forma de $\mathrm{NADH}_{2}$, debe ser transferido a un receptor de electrones para regenerar NAD+. En la fermentación alcohólica no es el ácido pirúvico sino su producto de descarboxilación, es decir el acetaldehído el que sirve de aceptor final de electrones ${ }^{4}$. El $\mathrm{NADH}_{2}$ proviene de la oxidación del 3-fosfogliceraldehído. Las dos reacciones correspondientes son interdependientes y constituyen una oxidoreducción (figura 1$)^{5}$.

Desde el punto de vista teórico 100 gramos de azúcares producen alrededor de 50 gramos de alcohol y 50 gramos de gas carbónico ${ }^{6}$. Además del alcohol etílico, en la fermentación alcohólica se producen a partir del ácido pirúvico otros compuestos, donde destacan el ácido acético, ácido butírico, acetona, ácido láctico, acetoína, butanodiol-2,3, ácido fumárico, ácido oxalacético, ácido succínico, ácido propiónico, diacetilo, etc 5 .

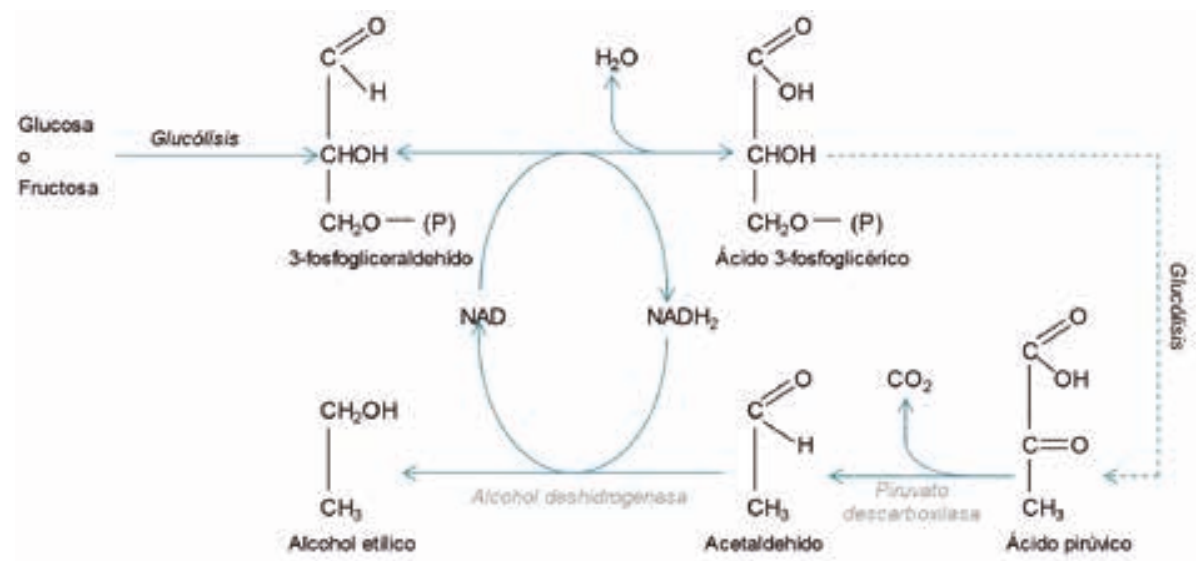

Figura 1. Esquema de la fermentación alcohólica.

La mayoría de los ácidos orgánicos restantes son producidos durante la vía de síntesis de los ácidos grasos a partir de la malonyl-Coenzima $\mathrm{A}^{8}$. Los ésteres se forman por reacciones entre alcoholes y ácidos, bajo la acción de esterasas de las levaduras y directamente por condensación del precursor del ácido acético, el acetil-CoA ${ }^{6}$.

La transformación de los aminoácidos de la uva, o los formados por la propia levadura, dan lugar a numerosos alcoholes superiores, que tienen más de dos átomos de carbono, tales como los alcoholes propílicos, butíricos o amílicos ${ }^{6}$. 


\section{Destilación}

Es una técnica usada para separar y seleccionar, mediante el uso del calor, componentes volátiles específicos a partir de una mezcla líquida (por ejemplo, el vino) ${ }^{9}$.

La elaboración de pisco será por destilación directa y discontinua, separando las cabezas y colas para seleccionar únicamente la fracción central del producto llamado cuerpo o corazón. Los equipos serán fabricados de cobre o estaño; se puede utilizar pailas de acero inoxidable ${ }^{1}$.

Los distintos compuestos destilados se pueden clasificar según la etapa en que destilan, tal como se muestra en la tabla $1^{10}$.

Algunas reacciones químicas tienen lugar en el curso de la destilación ${ }^{10}$ :

- La hidrólisis de diversos constituyentes del vino.

- Formación de acetato de etilo.

- Formación de furfural, durante todo el calentamiento sobre las pentosas.

- Fijación de ácidos grasos y sulfuros por el cobre.

- Formación de glicerol en las partes descendentes del alambique, a partir de las sustancias grasas por reacción sobre el cobre caliente.

\section{Componentes del pisco}

Se consideran componentes volátiles y congéneres del pisco, las siguientes sustancias: ésteres, furfural, ácido acético, aldehídos, alcoholes superiores y alcohol metílico ${ }^{1}$. Estos componentes están influenciados por varios factores durante el proceso de producción, entre los cuales podemos distinguir los siguientes ${ }^{11}$ :

1. Sustancias de aroma primario: generadas a partir de la variedad de la uva.

2. Sustancias de aroma secundario: generadas durante la vinificación y durante el proceso de fermentación.

3. Aromas de sustancias generadas durante el proceso de destilación.

4. Aromas de sustancias generadas durante el proceso de maduración o envejecimiento. 
Tabla 1. Etapa de destilación de algunos compuestos volátiles y su impacto en la calidad del pisco.

\begin{tabular}{|c|c|c|c|}
\hline $\begin{array}{l}\text { Compuestos } \\
\text { volátiles }\end{array}$ & De cabeza & De cuerpo & De cola \\
\hline Positivos & $\begin{array}{l}\text { Ésteres (acetato de hexilo, } \\
\text { acetato de 2-feniletilo, butirato } \\
\text { de etilo, hexanoato de etilo, } \\
\text { decanoato de etilo, } \\
\text { dodecanoato de etilo), terpenos } \\
\text { (linalol, óxidos de linalol y } \\
\text { nerol). }\end{array}$ & $\begin{array}{c}\text { Acetato de 3- } \\
\text { feniletilo, ternpenos } \\
\text { (hotrienol, a- } \\
\text { terpineol, linalol). }\end{array}$ & $\begin{array}{l}\text { Ésteres (lactato de } \\
\text { etilo, succinato de } \\
\text { dietilo), 2- } \\
\text { feniletanol. }\end{array}$ \\
\hline Negativos & $\begin{array}{l}\text { Aldehídos, acetato de etilo, } \\
\text { alcoholes superiores } \\
\text { (propanol, isobutanol, hexanol, } \\
\text { butanol, amílico, isoamílico), } \\
\text { homólogos saturados de } \\
\text { hexanol (cis-3-hexeno-1-ol, } \\
\text { trans-2-hexeno-1-ol, trans-3- } \\
\text { hexeno-1-ol), metanol. }\end{array}$ & $\begin{array}{l}\text { Ácido isobutírico, } \\
\text { metanol. }\end{array}$ & $\begin{array}{l}\text { Furfural, ácido } \\
\text { butírico, metanol. }\end{array}$ \\
\hline Neutros & - & - & $\begin{array}{l}\text { Ácido hexanoico, } \\
\text { ácido octanoico, } \\
\text { ácido decanoico. }\end{array}$ \\
\hline
\end{tabular}

\section{PARTE EXPERIMENTAL}

\section{Materiales y métodos}

\section{Materia prima}

Uva de la variedad Italia (Vitis vinífera L. var. Italia), procedente del fundo San Pablo en el distrito de Chincha Baja, provincia de Chincha, departamento de Ica.

\section{Análisis fisicoquímicos}

Determinación de componentes volátiles del pisco: metanol, furfural, acetaldehído, ácido acético, ésteres (acetato de etilo, acetato de isoamilo, formiato de etilo) y alcoholes superiores (propanol, isopropanol, butanol, isobutanol y alcohol iso/teramílico). Método cromatográfico según Norma Técnica Peruana NTP 211.03512.

\section{Análisis estadístico}

Los resultados se procesaron estadísticamente por análisis de varianza (ANOVA) y mediante la prueba de comparaciones múltiples de Tukey a un nivel de significancia de 5\%, procesados con el programa estadístico Minitab Release 14, para Windows. 


\section{Metodología experimental}

- Obtención del mosto: los racimos de uva Italia se procesaron en una prensa neumática para extraer el zumo y eliminar la piel y los racimos.

El mosto tuvo una densidad inicial de 1,098 g/L y una acidez de 3,0 g/L H2SO4.

- Fermentación: el mosto de uva Italia fue depositado en un tanque de acero inoxidable de 200 L con sistema de agitación y se agregó un pie de cuba con levadura seleccionada (levadura seca activa Saccharomyces cerevisiae Maurivin ${ }^{\mathrm{TM}}$ ).

Se introdujo el factor "nivel de fermentación", extrayendo muestras de vino base con diferentes tiempos de fermentación (previa homogeneización) en recipientes previamente desinfectados, expresados en cinco diferentes densidades de corte. Estas fueron: 1,040 $\mathrm{g} / \mathrm{L} ; 1,025 \mathrm{~g} / \mathrm{L} ; 1,010 \mathrm{~g} / \mathrm{L} ; 1,000 \mathrm{~g} / \mathrm{L}$ y $990 \mathrm{~g} / \mathrm{L}$, aproximadamente.

La utilización de levadura seca activa comercial es una práctica común entre las bodegas vitivinícolas como "Bodegas \& Viñedos Tabernero S.A.C.”. Tiene entre sus principales ventajas permitir un comienzo rápido de la fermentación ${ }^{13}$ e iniciar la fase exponencial de crecimiento en el menor tiempo posible ${ }^{14}$.

- Destilación: en un intervalo aproximado de 10 minutos desde la extracción de la muestra, se procedió a destilar aproximadamente $2.5 \mathrm{~L}$ de vino base en un alambique de cobre de $5 \mathrm{~L}$ de capacidad. Se separó al inicio de la destilación la primera fracción de destilado (cabeza) equivalente al $1 \%$ del volumen de vino a destilar y se cortó la misma a una graduación alcohólica de $42{ }^{\circ} \mathrm{G}$.L aproximadamente.

De acuerdo con Domenech ${ }^{15}$, en el Perú el porcentaje de cabeza eliminado (porcentaje al volumen del vino base a destilar) oscila entre 0,0 y $1,0 \%$. En el presente trabajo se ha considerado $1,0 \%$ por ser el valor más usado en los estudios de investigación de origen nacional. Con respecto a la graduación alcohólica final del pisco, según $\mathrm{Hatta}^{10}, 42^{\circ} \mathrm{G}$.L. es el grado alcohólico promedio de los piscos comerciales en el Perú.

- Conservación: los destilados se dejaron reposar durante tres meses en botellas de vidrio según lo recomendado por el Reglamento de la Denominación de Origen PISCO $^{1}$, antes de someterlos a análisis.

Luego de concluida esta etapa, los piscos fueron caracterizados determinando sus compuestos volátiles.

\section{Diseño experimental}

Para evaluar el factor "nivel de fermentación" del mosto, con sus niveles de densidad de corte: $1,040 \mathrm{~g} / \mathrm{L} ; 1,025 \mathrm{~g} / \mathrm{L} ; 1,010 \mathrm{~g} / \mathrm{L} ; 1,000$ g/L y $990 \mathrm{~g} / \mathrm{L}$; se utilizó un Diseño Completamente al Azar (DCA) con tres repeticiones.

\section{RESULTADOS Y DISCUSIÓN}

\section{Ácido acético}

Tal como se observa en la figura 2, el contenido de ácido acético tuvo menores concentraciones en los piscos provenientes de mostos con mayores niveles de fermentación. 
La utilización del ácido acético por las levaduras requiere primero una reducción a acetaldehído, favoreciendo la fermentación alcohólica en menoscabo de la pirúvica, con una disminución de la glicerina, pero con un aumento de los productos secundarios que pueden derivar del acetaldehído16. Es así que se observa una disminución del ácido acético a medida que aumenta el nivel de fermentación del mosto.

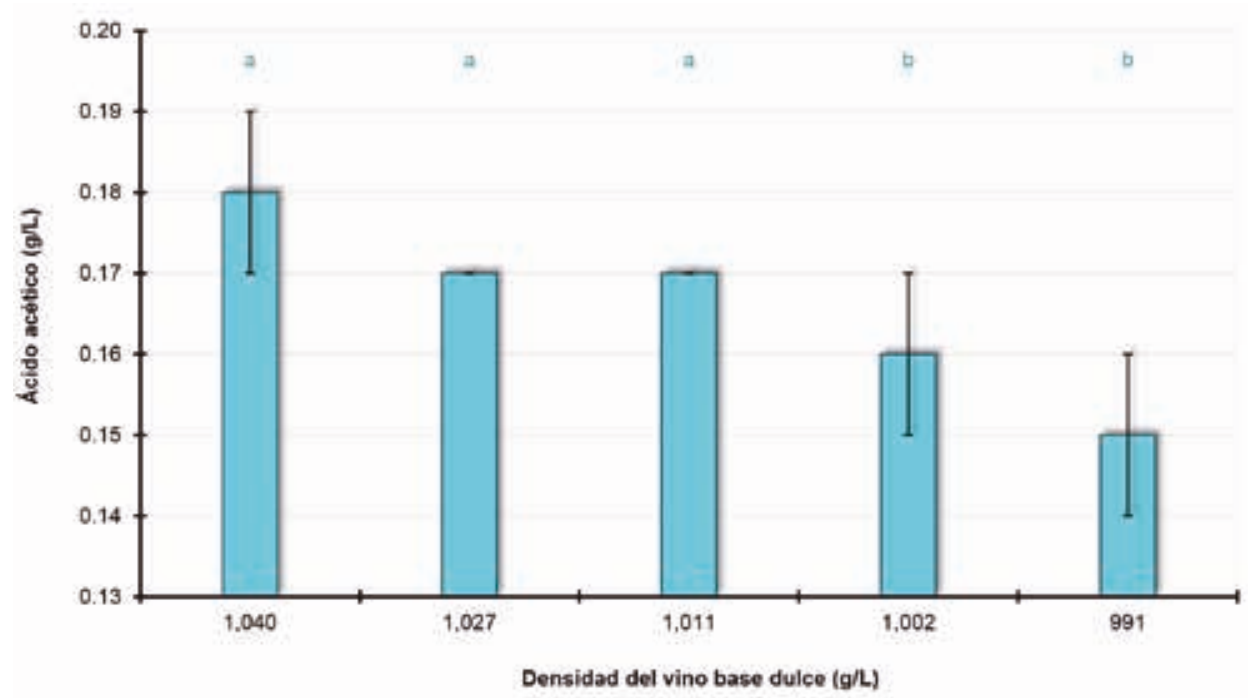

Figura 2. Contenido de ácido acético en los piscos provenientes de vinos base con diferentes niveles de fermentación.

Los contenidos promedio de ácido acético de los piscos en esta investigación, se encontraron por debajo del máximo permitido por el Reglamento de la Denominación de Origen PISCO (200 mg/100 ml A.A.) 1 , que es equivalente a $0,76 \mathrm{~g} / \mathrm{L}$.

\section{Propanol}

Tal como se observa en la figura 3, el contenido de propanol tuvo menores concentraciones en los piscos provenientes de mostos con mayores niveles de fermentación. La diferencia resultó significativa entre todos los tratamientos.

La formación de los alcoholes superiores en el curso de la fermentación alcohólica sucede linealmente con la producción de alcohol etílico para todos los alcoholes excepto para el propanol-1, que se forma fundamentalmente en las primeras fases de la fermentación ${ }^{16}$. En efecto, habiéndose realizado el muestreo de los mostos a partir de una densidad de 1,040 g/L (día 3 de la fermentación), no se esperaba un aumento en el contenido de propanol; ya que su biosíntesis tuvo lugar en los primeros días de la fermentación.

Una posible explicación del descenso en el contenido de propanol, a medida que aumenta el nivel de fermentación, podría haber sido la esterificación de este alcohol. Se ha encontrado 
en algunos vinos blancos el acetato de propilo, entre otros compuestos ${ }^{17}$. Del mismo modo, Conde et al. ${ }^{18}$ identificaron este mismo éster en vinos blancos elaborados con variedades Gual (Albilla), Malvasía y Verdello.

De acuerdo a los resultados estadísticos, habría que considerar que los piscos tipo Mosto Verde serán siempre significativamente mayores en cuanto al contenido de propanol que los provenientes de vinos base secos recién fermentados (figura 3 ).

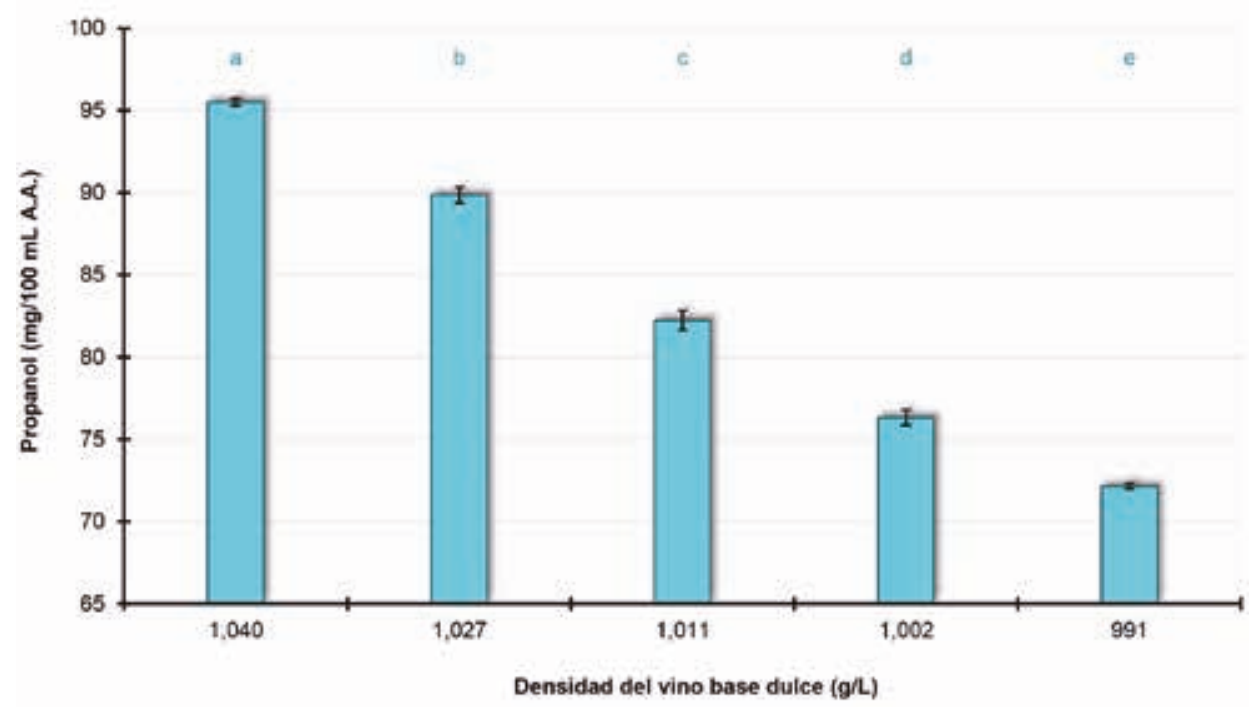

Figura 3. Contenido de propanol en los piscos provenientes de vinos base con diferentes niveles de fermentación.

De acuerdo con el Reglamento de la Denominación de Origen PISCO ${ }^{1}$, el propanol, entre otros alcoholes superiores, debe estar presente sin precisar exigencias de máximos y mínimos. Sin embargo, los contenidos promedio de propanol de los piscos en esta investigación, en especial los provenientes de mostos con 1,040 g/L de densidad (95,50 mg/100 mL A.A.), presentaron valores bastante altos.

El propanol y butanol, a concentraciones normales, son inodoros pero contribuyen a las características del alcohol mismo ${ }^{10}$. Es así, que concentraciones elevadas de propanol, como las que se detectaron en el presente estudio, podrían influir significativamente en las características sensoriales finales de los piscos.

\section{Alcohol iso/ter-amílico}

Tal como se observa en la figura 4, el contenido de alcohol iso/ter-amílico tuvo mayores concentraciones en los piscos provenientes de mostos con mayores niveles de fermentación. La diferencia resultó significativa entre todos los tratamientos. 
El contenido de alcohol iso/ter-amílico (mezcla de alcohol iso-amílico o metil-3-butanol-1 y alcohol ter-amílico o metil-2-butanol-2) en los piscos provenientes de mostos con diferentes niveles de fermentación, coincide con lo mencionado por Usseglio-Tomasset ${ }^{16}$, quien afirma que la formación de alcoholes superiores sucede linealmente con la producción de alcohol etílico para todos los alcoholes excepto para el propanol. Los alcoholes iso-amílico y feniletílico se forman en cantidades importantes durante la fermentación de los azúcares ${ }^{19}$.

De acuerdo a los resultados estadísticos, habría que considerar que los piscos tipo Mosto Verde serán siempre significativamente menores en cuanto al contenido de alcohol iso/teramílico que los provenientes de vinos base secos recientemente fermentados (figura 4).

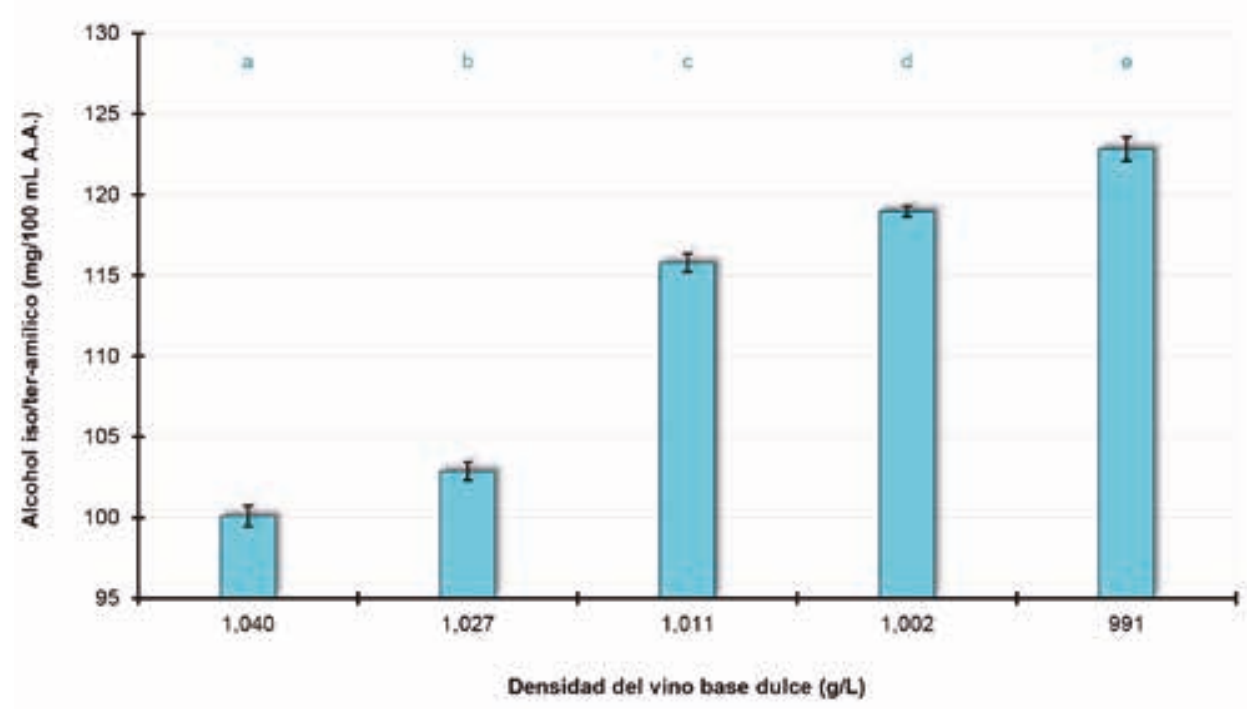

Figura 4. Contenido de alcohol iso/ter-amílico en los piscos provenientes de vinos base con diferentes niveles de fermentación.

\section{Acetato de etilo}

Tal como se observa en la figura 5, el contenido de acetato de etilo tuvo menores concentraciones en los piscos provenientes de mostos con mayores niveles de fermentación. La diferencia resultó significativa entre todos los tratamientos. 


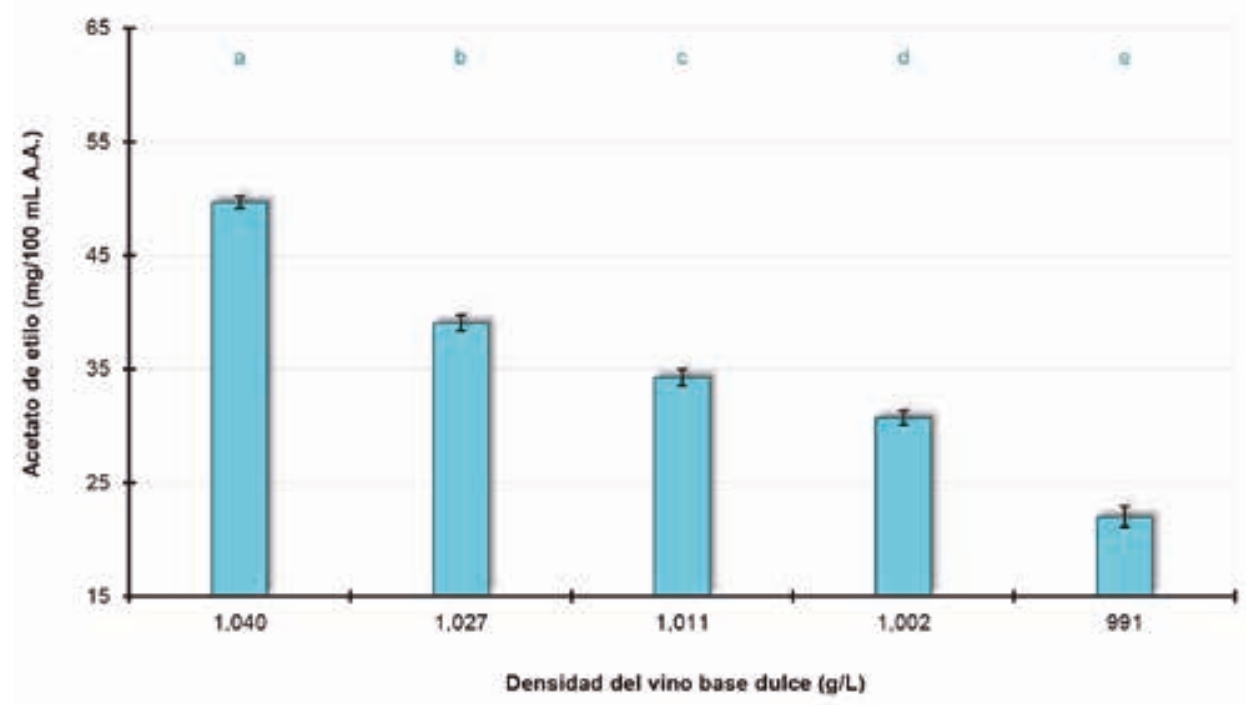

Figura 5. Contenido de acetato de etilo en los piscos provenientes de vinos base con diferentes niveles de fermentación.

Debido a las menores concentraciones de ácido acético en los piscos provenientes de vinos base con mayores niveles de fermentación, las concentraciones de acetato de etilo, al disminuir uno de sus sustratos, también fueron menores. De acuerdo con Vogt ${ }^{20}$, la formación del éster acético del etanol (acetato de etilo) tiene lugar por reacción del ácido acético con el alcohol etílico. Además, según Verstrepen et al. ${ }^{21}$, básicamente, son dos los factores más importantes para la velocidad en la producción de los ésteres: la concentración de los sustratos involucrados y el alcohol (etanol o alcoholes superiores).

De acuerdo a los resultados estadísticos, habría que considerar que los piscos tipo Mosto Verde serán siempre significativamente mayores en cuanto al contenido de acetato de etilo que los provenientes de vinos base secos recientemente fermentados (figura 5). Estos resultados coinciden con lo expuesto por Cacho et al. ${ }^{22}$, quien ha observado mayores niveles en la mayoría de ésteres y acetatos en los piscos tipo Mosto Verde, en comparación con piscos puros Italia.

Los contenidos promedio de acetato de etilo de los piscos en esta investigación, se encontraron entre el mínimo y el máximo permitido por el Reglamento de la Denominación de Origen PISCO (10,0 a 280,0 mg/100 mL A.A. $)^{1}$.

Aunque el acetato de etilo, cuantitativamente importante en el pisco, es considerado un componente negativo a la calidad por ser el responsable del olor a picado; las concentraciones encontradas en la mayoría de los piscos están por debajo del nivel de percepción ${ }^{10}$. Sin embargo, de acuerdo con Ribéreau-Gayon et al. ${ }^{4}$, el umbral de detección del acetato de etilo es cercano a $16 \mathrm{mg} / 100 \mathrm{~mL}$ A.A., por lo que en este caso, casi todos los valores obtenidos durante esta etapa sí afectarían negativamente el sabor y el olor del producto final. 


\section{Acetaldehído}

Tal como se observa en la figura 6, el contenido de acetaldehído tuvo menores concentraciones en los piscos provenientes de mostos con mayores niveles de fermentación. La diferencia resultó significativa entre todos los tratamientos.

La mayor parte de aldehídos se forman en la primera fase de la fermentación alcohólica, principalmente cuando los tratamientos prefermentativos son largos y la influencia del oxígeno es determinante ${ }^{23}$. El acetaldehído se acumula en esta etapa como consecuencia de la ausencia de la enzima alcohol deshidrogenasa de la levadura, la cual reduce el acetaldehído en alcohol ${ }^{23}$ (figura 1) y debido al período de inducción que conduce a la formación de glicerina ${ }^{24}$. En efecto, habiéndose realizado el muestreo de los vinos base a partir de una densidad de 1,040 g/L (día 3 de la fermentación), no se esperaba un aumento en el contenido de acetaldehído; ya que su biosíntesis habría tenido lugar en los primeros días de la fermentación.

La disminución en la concentración de acetaldehído que se observa en los piscos provenientes de vinos con mayores niveles de fermentación, se debe al consumo que sufre este compuesto durante la fermentación alcohólica. De acuerdo con Usseglio-Tomasset ${ }^{16}$, es el acetaldehído acumulado antes de la fermentación alcohólica, el que precisamente permite el inicio de la misma (figura 1).

De acuerdo a los resultados estadísticos, habría que considerar que los piscos tipo Mosto Verde serán siempre significativamente mayores en cuanto al contenido de acetaldehído que los provenientes de vinos base secos recientemente fermentados (figura 6).

Los contenidos promedio de acetaldehído en los piscos provenientes de mostos con 1,040 g/L de densidad (78,62 mg/100 mL A.A.), se encontraron por encima del máximo permitido por el Reglamento de la Denominación de Origen PISCO (3,0 a 60,0 mg/100 mL A.A.) ${ }^{1}$.

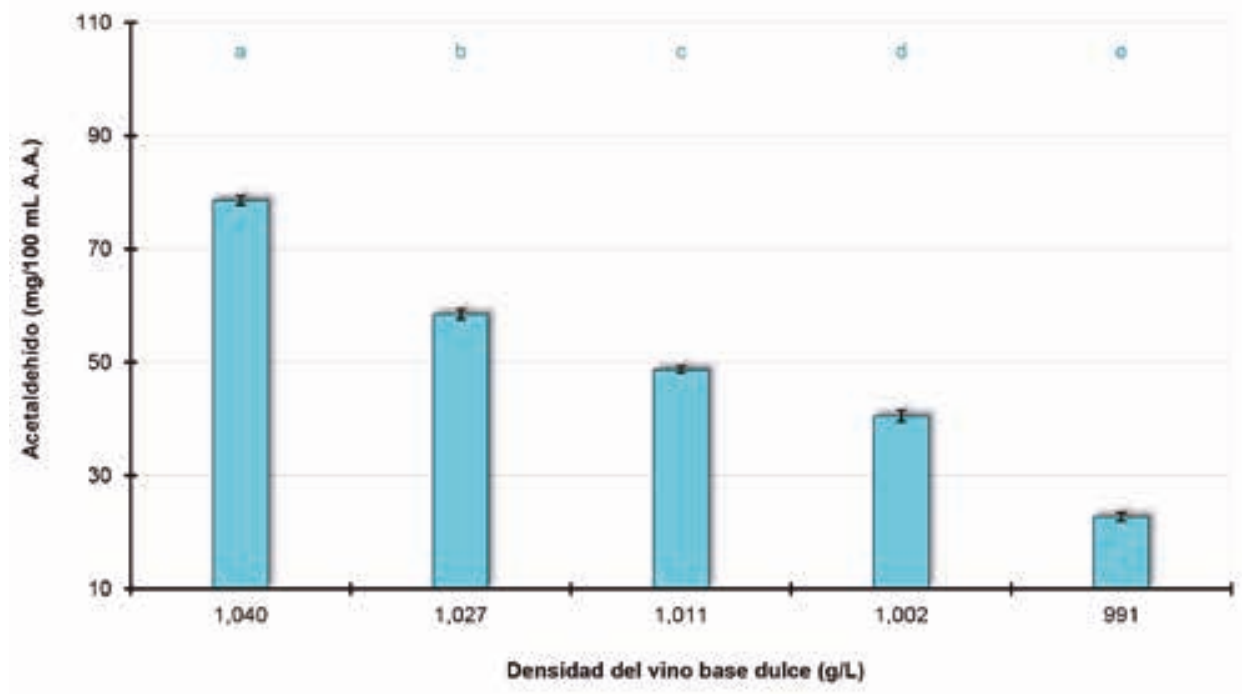

Figura 6. Contenido de acetaldehído en los piscos provenientes de vinos base con diferentes niveles de fermentación. 
Según Suárez ${ }^{25}$, el acetaldehído forma parte del aroma normal del vino pero en altas concentraciones ejerce un efecto desfavorable, participando directamente en el gusto a "oxidado". Es así, que concentraciones elevadas de acetaldehído como las que se detectaron en estos casos, podrían influir negativamente en las características sensoriales finales de los piscos.

\section{Metanol}

Tal como se observa en la figura 7, el contenido de metanol tuvo menores concentraciones en los piscos provenientes de mostos con mayores niveles de fermentación. La diferencia resultó significativa entre los cuatro primeros tratamientos.

Del mismo modo que para el propanol e iso-butanol, una posible explicación de las menores concentraciones de metanol en los piscos provenientes de vinos base con mayores niveles de fermentación, sería la esterificación de este alcohol. Salinas y Alonso ${ }^{18}$ han encontrado en algunos vinos blancos el acetato de metilo, entre otros compuestos.

De acuerdo a los resultados estadísticos, habría que considerar que los piscos tipo Mosto Verde con más de $1,010 \mathrm{~g} / \mathrm{L}$ de densidad, serán significativamente mayores en cuanto al contenido de metanol que los provenientes de vinos base secos recientemente fermentados (figura 7).

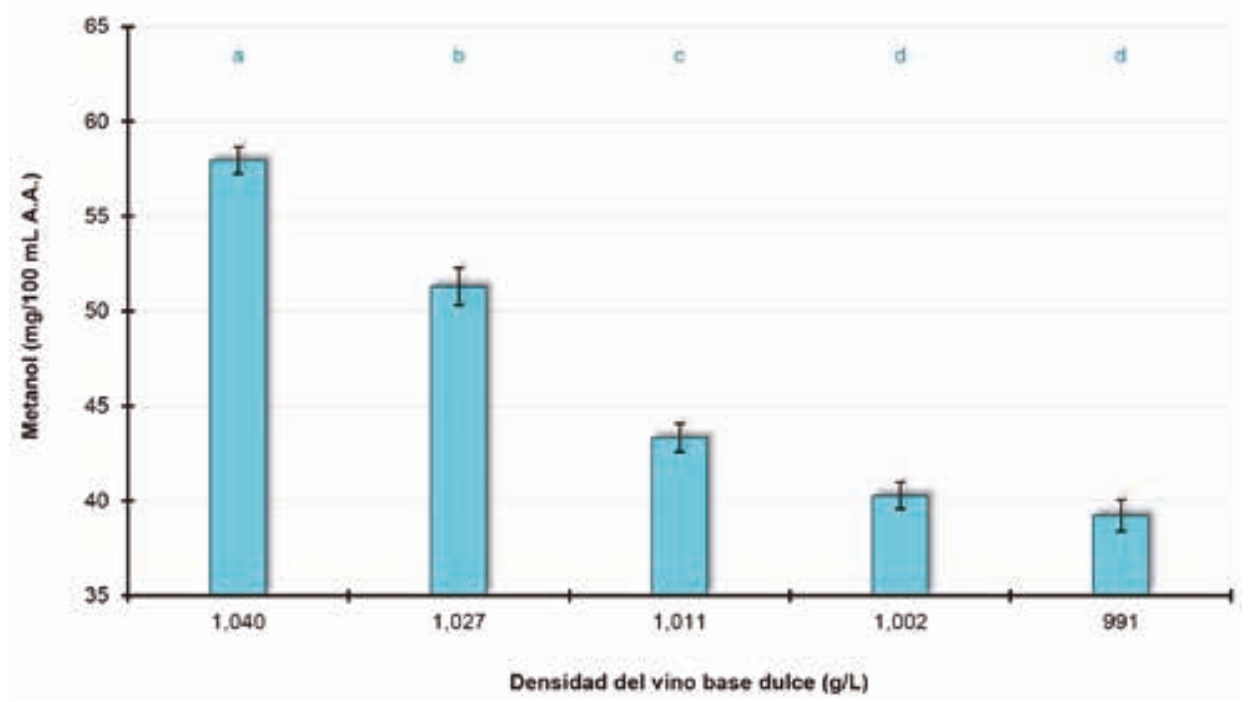

Figura 7. Contenido de acetaldehído en los piscos provenientes de vinos base con diferentes niveles de fermentación. 


\section{CONCLUSIONES}

Los piscos provenientes de vinos base con menores niveles de fermentación, expresados con mayores densidades de corte $(990 ; 1,000 ; 1,010 ; 1,025$ y 1,040 g/L), presentan mayores contenidos de ácido acético $(0.15,0.16,0.17,0.17$ y $0.18 \mathrm{~g} / \mathrm{L})$, propanol $(72.15,76.33,82.25$, 89.86 y $95.50 \mathrm{mg} / 100 \mathrm{~mL}$ A.A.), acetato de etilo (22.00, 30.72, 34.26, 39.06 y $49.68 \mathrm{mg} / 100$ mL A.A.), acetaldehído (22.71, 40.52, 48.69, 58.51 y $78.62 \mathrm{mg} / 100 \mathrm{~mL}$ A.A.) y metanol (39.22, 40.27, 43.33, 51.30 y $57.95 \mathrm{mg} / 100 \mathrm{~mL}$ A.A.); y menores contenidos de alcohol iso/ ter-amílico (122.83, 118.98, 115.78, 102.88 y 100.11 mg/100 mL A.A.).

\section{AGRADECIMIENTO}

A "Bodegas \& Viñedos Tabernero S.A.C." por las instalaciones, materia prima, insumos, equipos y materiales. A "La Molina Calidad Total Laboratorios" por los análisis de cromatografía de gases.

\section{REFERENCIAS BIBLIOGRÁFICAS}

1. Asociación Nacional de Productores de Pisco. Reglamento de la Denominación de Origen Pisco. Lima: INDECOPI; 2011.

2. INDECOPI. Norma Técnica Peruana: Bebidas alcohólicas. Pisco. Buenas prácticas de elaboración del pisco. NTP 212.034: 2007. Lima: INDECOPI; 2007.

3. Girard G. Bases científicas y tecnológicas de la enología. 1era edición. Zaragoza: Editorial Acribia S.A.; 2004.

4. Ribéreau-Gayon P, Glories Y, Maujean A, Dubourdieu D. Tratado de enología: química del vino. 1era edición. Buenos Aires: Editorial Hemisferio Sur S.A.; 2003.

5. Oreglia F. Enología teórico-práctica; volumen 1. 3era edición. Buenos Aires: Ediciones Instituto Salesiano de Artes Gráficas; 1978.

6. Blouin J, Peynaud É. Enología práctica: conocimiento y elaboración del vino. 4ta edición. Madrid: Ediciones Mundi-Prensa; 2004.

7. Hidalgo J. Tratado de enología; tomos I y II. 2da edición. Madrid: Ediciones MundiPrensa; 2011.

8. Flanzy C. Enología: fundamentos científicos y tecnológicos. 1era edición. Madrid: Vicente Ediciones y Ediciones Mundi-Prensa; 2000.

9. Léauté R. Distillation in alambic. Am J Enol Viticult. 1990; 41: 90-103.

10. Hatta B. Influencia de la fermentación con orujos en los componentes volátiles del pisco de uva Italia (Vítis vinífera L. var. Italia). $\square$ Tesis de maestría $\square$. Lima: Universidad Nacional Agraria La Molina; 2004.

11. Miličevič B, Banović M, Kovačevič K, Gracin, L. Impact of grape varieties on wine distillates flavour. Food Technol Biotech. 2002; 40: 227-232.

12. INDECOPI. Norma Técnica Peruana: Bebidas alcohólicas. Método de ensayo. Determinación de metanol y de congéneres en bebidas alcohólicas y alcohol etílico 
empleado en su elaboración, mediante cromatografía de gases. NTP 211.035: 2008. Lima: INDECOPI; 2008.

13. Rankine B. Manual práctico de enología. 3era edición. Zaragoza: Editorial Acribia S.A.; 2000 .

14. Asencios E. Influencia de la fermentación con levaduras nativas y comerciales en las características del pisco de Uvina o Jacquez (Vitis aestivalis-cinerea x Vitis vinífera) del valle de Lunahuaná. [Tesis de pregrado]. Lima: Universidad Nacional Agraria La Molina; 2007.

15. Domenech A. Influencia de la maceración con orujos y corte de cabeza en el contenido de terpenos en piscos de la variedad Italia (Vítis vinífera L. var. Italia). [Tesis de pregrado]. Lima: Universidad Nacional Agraria La Molina; 2006.

16. Usseglio-Tomasset L. Química enológica. 4ta edición. Madrid: Ediciones MundiPrensa; 1998.

17. Salinas MR, Alonso G. La destilación a presión reducida como método de separación de componentes aromáticos mayoritarios de mostos y vinos. Rev Fac Educ Univ Albacete. 1988; 2: 217-222.

18. Conde JE, Rodríguez JJ, Cabrera HM, Pérez SJ, Ferreira V, Cacho JF. Compuestos volátiles en vinos blancos elaborados con las variedades Gual, Malvasía y Verdello. Paper presentado en: Jornadas Técnicas Vitivinícolas Canarias; 2003; Zaragoza-España.

19. Bulnes L, Gisseleire A. Bases para la determinación de autenticidad del pisco chileno mediante cromatografía de gases. [Tesis de pregrado]. Santiago: Pontificia Universidad Católica de Chile; 1983.

20. Vogt E. Fabricación de vinos. 1era edición. Zaragoza: Editorial Acribia; 1972.

21. Verstrepen K, Derdelinckx G, Dufour J-P, Winderickx J, Thevelein J, Pretorius I, Delvaux F. Flavor-active esters: adding fruitiness to beer. J Biosci Bioeng. 2003; 96: 110-118.

22. Cacho JF, Moncayo L, Palma JC, Ferreira V, Culleré L. Informe del estudio realizado para caracterizar diferentes piscos peruanos. Aragón: Instituto de Investigación en Ingeniería de Aragón; 2012.

23. Wondra M, Berovič M. Analyses of aroma contents of Chardonnay wine fermented by different yeast strains. Food Technol Biotech. 2001; 39: 141-148.

24. Aleixandre JL, Álvarez MI. Tecnología enológica. 1era edición. Madrid: Editorial Síntesis; 2003.

25. Suárez JA. Levaduras vínicas: funcionalidad y uso en bodegas. 1era edición. Madrid: Ediciones Mundi-Prensa; 1997. 